Rev. Elev. Méd. vét. Pays trop., 1972, 25 (2) : 245-257

\title{
Extériorisation des potentialités du zebu Gobra Résultats des abattages pratiqués en 1971
}

\author{
par J.-P. DENIS (*), J. VALENZA $(* *)$ et A. I. THIONGANE (***)
}

\section{RESUME}

Ces nouvelles observations, qui portent sur l'étude approfondie des carcasses de jeunes zébus mâles, entretenus depuis toujours en embouche intensive, suivant des modalités alimentaires et des durées différentes, confirment le potentiel considérable des animaux de cette race, pour la production de viande.

\section{INTRODUCTION}

L'expérience d'extériorisation des potentialités génétiques du zébu Gobra se poursuit depuis 1968. Les résultats de la croissance et des premiers abattages ont déjà été rapportés (DENIS et VALENZA, 1971) (4).

Le présent travail se propose de rendre compte des abattages effectués en octobre 1971.

\section{MATERIEL ET METHODES}

\section{Animaux expérimentaux}

Six animaux ont été abattus. Trois proviennent du lot de mâles entretenu depuis 1968 avec une alimentation à volonté. Les trois autres sont de jeunes animaux issus du lot de femelles extériorisées.

En effet, depuis janvier 1970 , les femelles de ce lot ont été placées en compagnie d'un mâle dans le but d'étudier l'influence de l'alimentation sur les qualités de reproductrices et

(*) Section de Recherches I.E.M.V.T. du Centre de Recherches zootechniques de Dahra-Djoloff.

(**) Laboratoire national de l'Elevage et de $\mathbf{R e}$ cherches vétérinaires, B.P. $\pi^{\circ}$ 2057, Dakar.

(***) Directeur du Centre de Recherches zootechniques de Dahra-Djoloff. de nourrices des femelles Gobra. Un compte rendu détaillé de cette expérience sera publié ultérieurement. Ces trois jeunes mâles ont, par conséquent, bénéficié d'une alimentation distribuée à volonté comme leurs prédécesseurs mais aussi de conditions alimentaires très améliorées de leurs mères.

L'abattage a été effectué à l'abattoir de Dakar après un jeûne de 43 heures et un voyage de $270 \mathrm{~km}$ en bétaillère.

\section{Alimentation}

Les animaux ont continué à recevoir, en plus de pâturage naturel mis à leur disposition, un concentré distribué à volonté. Mais la composition de ce dernier a été modifiée par rapport à celui distribué jusqu'alors (janvier 1971).

Cette composition est la suivante (en $\mathrm{kg}$ ) : Maïs . . . . . . . . 10

Son de blé . . . . . . . . 40

Son de maïs . . . . . . . 17

Son de sorgho. . . . . . . . 29,50

Tourteau d'arachide . . . . . 0,50

Carbonate de calcium . . . 2,25

Chlorure de sodium . . . . 0,50

Complément vitaminé . . . . 0,25

Le concentré distribué est donc moins riche : $0,85 \mathrm{UF}$ et $100 \mathrm{~g}$ de M.A.D. par $\mathrm{kg}$ au lieu 
de $0,90 \mathrm{UF}$ et $125-130 \mathrm{~g}$ de M.A.D. par $\mathrm{kg}$.

Il faut signaler, à propos du pâturage naturel, que celui-ci a été pratiquement inexistant à la suite de l'hivernage 1970 catastrophique. En effet, la quantité d'eau enregistrée n'a été que de $210 \mathrm{~mm}$, alors que le C.R.Z. de Dahra se trouve sur l'isohyète $520 \mathrm{~mm}$. Pareil déficit n'avait pas été constaté depuis 1941 (205 mm).

\section{Observations}

Les observations: pesées et mensurations classiques ont continué à être effectuées, mais le rythme est devenu moins rapide: tous les 3 mois au lieu de tous les mois comme jusqu'à 24 mois.
RESULTATS

\section{ANIMAUX NES EN 1968}

\subsection{Croissance pondérale}

De 24 à 36 mois, le gain de poids moyen journalier a été de $511 \mathrm{~g}$ chez les mâles. Chez les femelles, le gain de poids journalier ne signifie plus grand chose étant donné les épisodes gravidiques qu'elles subissent depuis janvier 1970, c'est-à-dire depuis l'âge de 19 mois environ.

Les poids moyens seront par contre donnés pour les deux sexes. Les témoins sont toujours constitués par les animaux nés au cours des années 1965-66-67 (tableau $\mathrm{n}^{\circ} \mathrm{I}$ ).

TABLEAU $\mathrm{N}^{\mathrm{D}} \mathrm{I}$

\begin{tabular}{|c|c|c|c|c|c|}
\hline \multirow{3}{*}{} & & \multicolumn{2}{|c|}{ Extêriorisation } & \multicolumn{2}{c|}{ Têmoins (5) } \\
\cline { 2 - 6 } & & 24 mois & 36 mois & 24 mois & 36 mois \\
\hline \multirow{3}{*}{ Mâles } & Nombre & 14 & 10 & 95 & 65 \\
& Moyenne & 490,0 & 634,2 & 296,6 & 364,3 \\
& \pm & 24,2 & 25,5 & 7,8 & 13,3 \\
\hline \multirow{3}{*}{ Femelles } & Nombre & 28 & 25 & 110 & 89 \\
& Moyenne & 387,8 & 42,6 & 220,4 & 309,5 \\
& \pm & 15,6 & 20,4 & 7,4 & 9,2 \\
\hline
\end{tabular}

I] faut signaler que la diminution de la vitesse de croissance rencontrée en fin de saison sèche entre le $21^{\mathrm{e}}$ et $24^{\mathrm{e}}$ mois a été constatée de même entre le $33^{\mathrm{e}}$ et le $36^{\mathrm{e}}$ mois. L'extrême pauvreté des pâturages en fin de saison sèche, particulièrement en 1971, n'est probablement pas étrangère à ce fait.

\subsection{Abattages}

Les données générales concernant les 3 animaux abattus le 27 octobre sont présentées au tableau $n^{\circ}$ II et les résultats de l'abattage apparaissent au tableau $\mathrm{n}^{0}$ III.

TABLEAU $N^{\circ} \mathrm{II}$

\begin{tabular}{|c|c|c|c|c|c|c|}
\hline $\mathrm{N}^{\mathrm{D}}$ & $\begin{array}{c}\text { Date } \\
\text { naissance }\end{array}$ & $\begin{array}{c}\text { Poids } \\
\text { naissance }\end{array}$ & $\begin{array}{c}\text { Poids } \\
\text { a } 36 \text { mois }\end{array}$ & $\begin{array}{c}\text { Poids vif et } \\
\text { (âge) abattage }\end{array}$ & $\begin{array}{c}\text { Gain de poids } \\
\text { total } 0 \text {-36 mois }\end{array}$ & $\begin{array}{c}\text { Gain de poids } \\
\text { jour }\end{array}$ \\
\hline 2733 & 23.6 .68 & 19 & 676 & $663(40 \mathrm{~m})$ & 657 & 0,600 \\
2749 & 22.7 .68 & 25 & 651 & $639(39 \mathrm{~m})$ & 626 & 0,490 \\
2741 & 26.8 .68 & 18 & 550 & $542(38 \mathrm{~m})$ & 532 & 0,570 \\
\hline
\end{tabular}


Poids avant jeûne

Poids après jeûne

Pourcentage de perte au jeûne

Poids carcasse chaude

Poids carcasse froide

Pourcentage de perte au ressuyage

Rendement

Rendement vrai

Pourcentage contenu de panse

Pourcentage 5ème quartier

En pourcentage du poids de la carcasse

froide : (G)

- épaule

- pis

- panneau

- train de côtes

- globe

- bosse

- gras de rognon

- cuir

- queve

Longueur carcasse

Epaisseur cuisse

Epaisseur plat de côtes

Indice de compacité

Conformation carcasse

Gras carcasse

Graisse de couverture

Gras de rogron

Graisse musculaire

Indice de gras

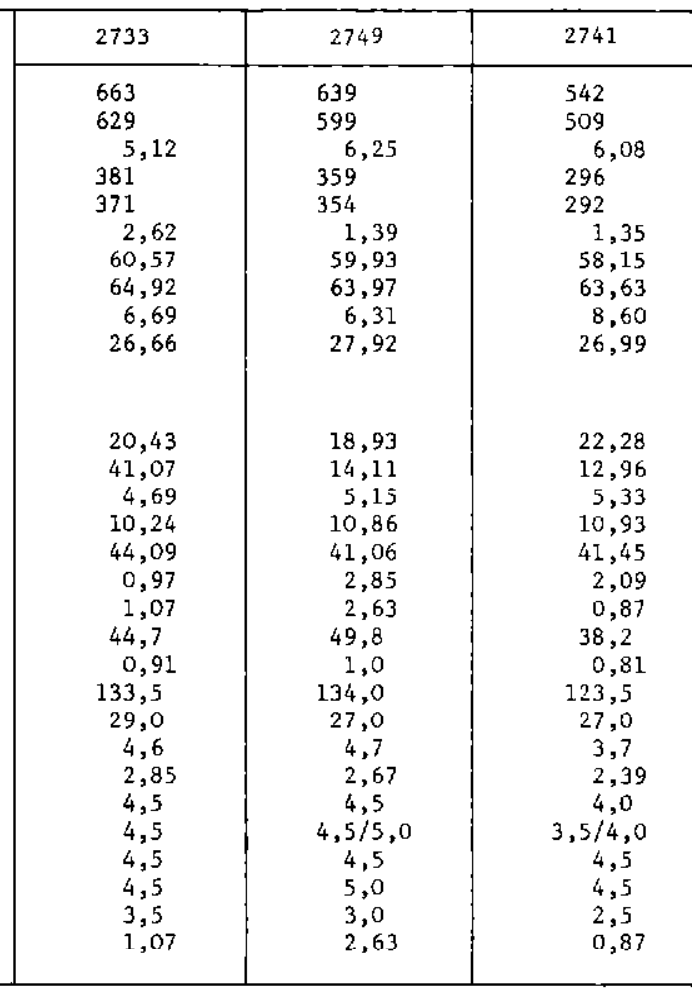

\section{Commentaires}

1. Le pourcentage de pertes au jeûne, qui avait été mal évalué en 1970, montre des valeurs classiques.

2. La perte au ressuyage est faible et comparable à celle observée en 1970. Encore une fois, la graisse de couverture en bonne quantité a empêché des pertes trop importantes par dessiccation.

3. Les rendements restent très bons, mais cependant moins élevés qu'en 1970 (59,6 p. 100 en moyenne contre 63,7 p. 100). Ces rendements restent cependant comparables à ceux des jeunes bœufs gras du marché français ( 60 p. 100 de rendement en moyenne).

4. Le pourcentage de contenu de panse est légèrement plus élevé qu'en 1970, mais reste assez faible. L'aliment, presque exclusivement à base de concentré, consommé par les animaux en est la raison.

5. L'indice de gras est peu élevé par rapport à 1970 (indice moyen 1,52 contre 2,83).

6. La viande a une couleur correcte de même que la graisse qui est claire. Ces constata- tions avaient aussi été faites lors des abattages de 1970 . Le persillé et le marbré sont présents également.

7. Dans l'ensemble donc, les carcasses des animaux abattus en 1971 ont les mêmes caractéristiques principales que les précédentes. Le pourcentage de gras est cependant plus faible. Les performances générales restent très au-delà de celles observées sur le marché traditionnel.

\section{Découpe anatomique}

En 1970 , lors des premiers abattages, le travers "excès de gras" des animaux avait été apprécié d'une part, d'une manière subjective à l'examen des carcasses, d'autre part, en calculant l'indice de gras: celui-ci êtait élevé surtout pour l'une des carcasses : 1,94. $2,29-4,26$.

Il a semblé intéressant, en 1971, de procéder à une analyse plus détaillée de l'une des carcasses pour déterminer les rapports existant entre carcasse, viande, gras et os $\left(n^{\circ} 2733\right.$ demicarcasse droite).

Cependant, on a pu observer que l'indice de gras en 1971 est tout à fait convenable pour 
deux carcasses et un peu élevé pour la troisième $(2,63)$.

La découpe a été faite muscle par muscle en séparant les os et la graisse divisée en graisse de couverture, graisse interne et graisse intermusculaire.

Les résultats sur le plan des poids musculaires sont présentés sous deux formes:

- l'ensemble des muscles isolés sur la carcasse apparaît aux résultats I. Il faut souli- gner que les muscles du cou ne sont pas présents en totalité puisque les prélèvements coutumiers se situent essentiellement à ce niveau. Par conséquent, seuls les muscles de la base du cou sont présents, soit entiers soit le plus souvent tronqués;

- la deuxième présentation fait apparaître les différents groupes régionaux musculaires donnant les traits essentiels de la carcasse. Les muscles importants sur le plan des comparaisons sont cependant restés isolés (résultats II).

\subsection{Muscles}

\section{RESULTATS I}

Découpe muscle par muscle de la demi-carcasse droite

de lanimal $n^{o} 2733$

La nomenclature utilisée est tirée de Nomina anatomica veterinaria, 1968, Vienne (International comittee on veterinary anatomical nomenclature. World association of Veterinary anatomists).

1. Membre thoracique

1.1. Epaule

— Deltoïde $(M$. deltoïdus $)$. . . . . . . . . . . . . . . . . $0,0,420$

- Sus-épineux (M. Supra spinatus) . . . . . . . . . . . . . . . . $1,01,650$

— Sous-épineux (M. infra spinatus) . . . . . . . . . . . . . 2,000

- Sous-scapulaire $(M . s u b$ scapularis) . . . . . . . . . . . . . . . . $\quad 1,000$

- Coraco brachial (M. coraco brachialis) . . . . . . . . . . . . . 0,120

- Angulaire de l'épaule (M. serratus ventralis cervicis) . . . . . . . . $\quad 3,700$

1.2. Bras

- Biceps brachial (Biceps brachil) . . . . . . . . . . . . $0,0,700$

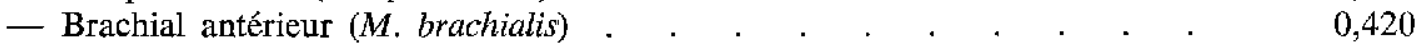

- Triceps brachial ( $M$. triceps brachii) . . . . . . . . . . . . 4,830

1.3. Avant-bras

- Extenseurs des phalanges (antérieur et latéral) (M. $m$ extensor digitorum communis et extensor digitorum lateralis) . . . . . . . . . . . .

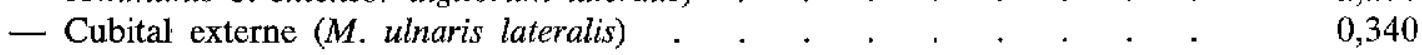

- Cubital interne $(M$. flexor carpi ulnaris) . . . . . . . . . . . . . . $\quad$. $\quad 0,610$

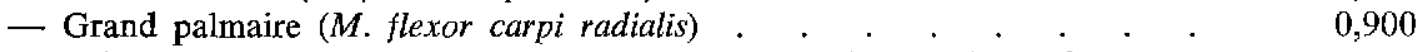

- Fléchisseurs des phalanges (superficiel et profond) (M. $m$ flexor digitorum

2. Muscles du bassin

2.1. Région fessière

- Fessier moyen $(M$. gluteus medius) . . . . . . . . . . . . . . . . 3,450

- Fessier accessoire (M. gluteus accessorius) . . . . . . . . . . . . . $0,0,200$

- Fessier profond (M. gluteus profondus) . . . . . . . . . . . . 0,410

2.2. Région pelvicrurale profonde

- Obturateur externe et portion intrapelvienne ( $M$. obturatorius externus pars externa - pars intrapelvina) 
3. Muscles de la cuisse

3.1. Région crurale interne

- Couturier (M. sartorius)

- Droit interne ( $M$. gracilis) .

- Pectiné (M. pectineus) .

- Adducteurs de la cuisse (plan profond) ( $M . m$ adductores)

3.2. Région crurale antérieure

- Tenseur du fascia lata ( $M$. tensor fasciae latae).

- Droit antérieur ( $M$. rectus femoris)

- Vaste externe ( $M$. vastus lateralis)

- Vaste interne ( $M$. vastus medialis)

(M. quadriceps femorus)

3.3. Région crurale postérieure

- Long vaste (paraméral et biceps fémoral) (M. $m$ gluteo biceps et biceps femoris)

— Demi-tendineux (M. semitendinosus) . . . . . . . . .

- Demi-membraneux (M. semimembranosus) $\cdot$. . . . . . . . .

3.4. Muscles de la jambe (M. m flexor digitorus superficialis, Popliteus, Flexor digitorum profondis) .

- Gastrocnémien (+ Soleare) (M. triceps surae)

4. Muscles du tronc

\subsection{Peaucier}

- Thoraco abdominal ( $M$. cutaneus tronci)

4.2. Muscles rachidiens spinaux-dorso-lombaires

- Long costal (M. ilio costalis lomborum et thoracis)

- Long dorsal (M. longissimus - lomborum et thoracis)

- Long épineux ( $M$. spinalis thoracis)

- Muscles du noyau vertébral du cou et muscles du cou $(M, m$ musculii colii - C 3 à $\mathrm{C} 7$ ).

5. Muscles du thorax

5.1. Région dorso-spinale

- Portion dorsale du trapèze ( $M$. trapezius - pars thoracica)

- Grand dorsal (M. latessimus dorsi) .

- Petit dentelé oral (M. seratus dorsalis caudalis et cranialis)

5.2. Région costale

- Grand dentelé (M. seratus ventralis thoracis) . . . . . . . . 1,000

- Inter costaux internes et externes (M. m intercostales externi et interni) . 3,030

5.3. Pectoraux (M. m pectorales superficiales et profondus) . . . . . . 4,380

6. Abdomen

6.1. Région abdominale inférieure

- Oblique externe ( $M$. obliquus externus abdominis)

- Oblique interne ( $M$. obliquus internus abdominis).

- Droit de l'abdomen ( $M$. rectus abdominus) .

- Transverse de l'abdomen ( $M$. transversus abdominis)

6.2. Région sous-lombaire

- Petit psoas (M. psoas minor)

- Grand psoas ( $M$. psoas major)

- Iliaque (M. iliacus)

- Carré des lombes ( $M$. quadratus lomborum)

- Intertransversaires des lombes ( $M . m$ intertrasversarii lomborum)

7. Bosse + Romboïde ( $M$. rhomboïdeus servicis $+M$. trapezius - pars cervicalis) 


\section{RESULTATS II}

Groupes régionaux musculaires

Membre thoracique

- Epaule

-. Bras

- Avant-bras

$3,120=17,960$

Membre pelvien

- Cuisse :

Région crurale interne

Région crurale antérieure

6,550

Région crurale postérieure

16,340

(demi-membraneux : 6,340)

- Jambe

$5,380=30,190$

Muscles du tronc

- Peaucier

- Muscles rachidiens spinaux dorso-lombaires + muscles du cou

- Long dorsal

$.6,560+28,940$
$.5,250=34,190$

- Bosse + rhomboïde

Muscles du bassin

- Région fessière

- Région pelvi-crurale profonde

Muscles du thorax

- Région dorso-spinale .

5,620

- Région costale

-. Pectoraux

$4,380=14,030$

\section{Abdomen}

- Région abdominale inférieure

— Région sous-lombaire . . . . . . . . . . . . . 3,740

Filet : petit psoas . . . . . . . . 0,200

grand psoas . . . . . . . . . 1,540

iliaque . . . . . . . . . . . 0,500

carré des lombes . . . . . . . . 0,800

intertransversaires des lombes . . . . 0,700

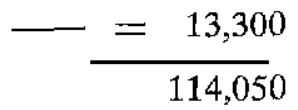

1.2.2. Os

1.2. 3. Graisse

- de couverture

- interne . . . . . . . 1,300

— musculaire . . . . . . . . . . 10,600

- gras de rognon . . . . . . . . . . . . . . 
La récapitulation des données de la découpe est la suivante :

Muscles . . . . . . . $114.050 \mathrm{~kg}$

Os . . . . . . . . $27,800 \mathrm{~kg}$

Graisse

$35,450 \mathrm{~kg}$
Soit en pourcentage par rapport au poids total de carcasse :

Muscles

Os
64,30 p. 100

15,67 p. 100

19,98 p. 100
Ce qui constitue pratiquement 35,65 p. 100 de déchets. Il faut noter de plus que les tendons et les aponévroses n'ont pas été systématiquement séparés des muscles. Dans le but de comparer la valeur des carcasses des zébus Gobra avec d'autres animaux de races différentes, divers résultats ont été regroupés et présentés au tableau $\mathbf{n}^{\circ}$ IV.

TABLEAU $N^{\circ}$ IV

\begin{tabular}{|c|c|c|c|c|}
\hline $\mathrm{R} a \mathrm{c} \mathrm{e}$ & $\begin{array}{l}\text { Pourcentage } \\
\text { de muscle }\end{array}$ & $\begin{array}{c}\text { Pourcentage } \\
\text { de gras }\end{array}$ & $\begin{array}{l}\text { Pourcentage } \\
\text { d'os }\end{array}$ & Auteurs \\
\hline $\begin{array}{l}\text { Hereford } \\
\text { Angus } \\
\text { Brahman } \\
\text { Croigês Brahman } \\
\text { Santa Gertrudis } \\
\text { Holstein } \\
\text { Jersey }\end{array}$ & $\begin{array}{l}54,5 \\
52,7 \\
60,1 \\
58,3 \\
57,1 \\
60,1 \\
57,0\end{array}$ & $\begin{array}{l}31,3 \\
34,3 \\
24,4 \\
26,9 \\
27,1 \\
22,1 \\
26,2\end{array}$ & $\begin{array}{l}14,1 \\
12,8 \\
15,3 \\
15,0 \\
15,7 \\
17,6 \\
16,2\end{array}$ & $\begin{array}{l}\text { (citës par J. Gilibert, } \\
\text { 1965) } \\
\text { Cole, Ramsey } \\
\text { Hobbes et Temple } \\
\text { (1964) }\end{array}$ \\
\hline \multirow[t]{2}{*}{$\begin{array}{l}\text { Frisons } \\
\text { Hereford } \\
\text { Shorthorn } \\
\text { Aberdeen Angus } \\
\text { Shorthorn }\end{array}$} & $\begin{array}{l}71,1 \\
70,7 \\
65,8 \\
67,7 \\
67,0 \\
65,3\end{array}$ & $\begin{array}{l}13,6 \\
15,0 \\
11,5 \\
12,7 \\
11,9 \\
11,6\end{array}$ & $\begin{array}{l}15,3 \\
14,1 \\
22,8 \\
19,4 \\
21,1 \\
23,4\end{array}$ & $\begin{array}{l}\text { Harte F.J. et } \\
\text { Conniffe D } \\
(1971)\end{array}$ \\
\hline & 70,08 & $\begin{array}{c}+ \text { aponévroses } \\
13,98 \\
(10,80+3,18)\end{array}$ & 15,94 & $\begin{array}{l}\text { Craplet C. (1966) } \\
\text { (école supérieure des } \\
\text { métiers de la viande) }\end{array}$ \\
\hline $\begin{array}{l}\text { Brune des Alpes } \\
\text { Modica Sardinian }\end{array}$ & 55,42 & $\begin{array}{l}+ \text { tendons } \\
23,61\end{array}$ & 20,97 & Rossi G. (1967) \\
\hline German Simmental & 71,25 & $\begin{array}{l}+ \text { tendors } \\
+10,08 \\
+\quad 3,26\end{array}$ & 15,4 & $\begin{array}{l}\text { Schmitter W. } \\
\text { Averdunk G;, Matzke D. } \\
\text { Pilzweger J., Rupp K. } \\
\text { Mittelstädt (1971) }\end{array}$ \\
\hline $\begin{array}{l}\text { Charolais } \\
-2 \text { a } 3 \text { ans } \\
\text { - vaches de réforme }\end{array}$ & 80 à 84 & - & - & Herd Book Charolais \\
\hline
\end{tabular}

Ces carcasses de zébus sénégalais présentent donc des proportions de muscles, gras et os relativement correctes. Par rapport aux bovins français, la proportion de graisse reste encore très importante mais celle-ci reste faible par rapport à celle rencontrée dans certaines races anglaises.

$\mathrm{Ce}$ qu'il faut noter c'est que d'une manière subjective, les carcasses engraissées normalement peuvent paraître extrêmement grasses à des personnes n'ayant l'habitude de ne voir qu'une majorité de carcasses dont la teneur en graisse est extrêmement faible, ce qui est le cas sur le marché de Dakar. De plus, il faut signaler que toute croissance très importante des bovins s'accompagne obligatoirement, sauf chez les Charolais en particulier, d'un certain accroissement des dépôts graisseux et, que de toute façon, pour avoir le maximum de viande maigre, il faut atteindre un poids maximal de carcasse (corrélation : 0,94 DUBOSE, CARTWRIGHT et COOPER, 1967). 


\section{ANIMAUX NES EN 1970}

\subsection{Evolution pondérale de Ia naissance à 12 mois}

TABLEAU N $\mathrm{N}^{\circ} \mathrm{V}$

Evolution pondérale comparëe des animaux extériarisés nés en 1968 et 1970 et des animaux tëmoing nës en 1965-66-67-68.

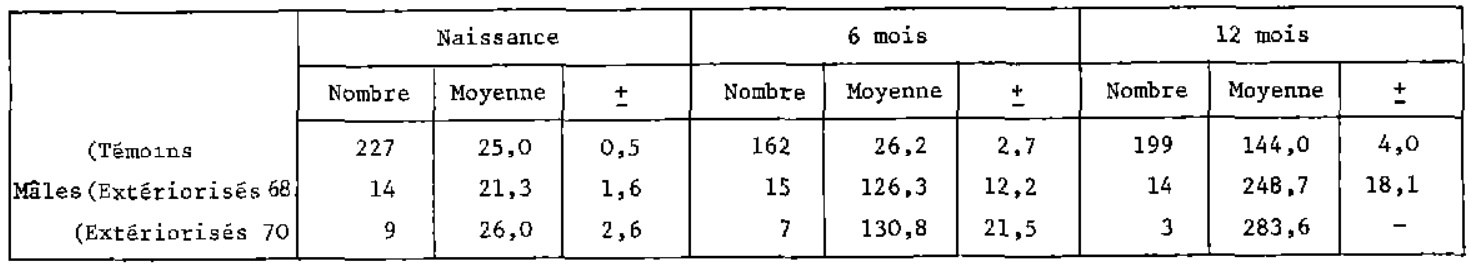

A la lecture du tableau $n^{\circ} \mathrm{V}$, il apparaît que les possibilités de croissance du zébu Gobra étaient encore limitées dans la première partie de l'expérience (animaux nés en 1968) à cause des conditions d'entretien non améliorées des mères. Ce qu'il est essentiel de retenir ici, ce sont les caractéristiques générales des trois animaux abattus à l'âge de 11 mois.

\subsection{Résultats des abattages}

Les données générales concernant les trois animaux apparaissent au tableau $\mathrm{n}^{0}$ VI.

TABLEAU $\mathrm{N}^{\circ} \mathrm{VI}$

\begin{tabular}{|c|c|c|c|c|c|c|}
\hline \multirow{2}{*}{$\mathrm{N}^{0}$} & $\begin{array}{c}\text { Date } \\
\text { naissance }\end{array}$ & $\begin{array}{c}\text { Poids } \\
\text { naissance }\end{array}$ & a 10 mois & $\begin{array}{c}\text { Poids vif et } \\
\text { (âge) abattage }\end{array}$ & $\begin{array}{c}\text { Gain de poids } \\
\text { total 0-10 mois }\end{array}$ & $\begin{array}{c}\text { Gain de poids/ } \\
\text { jour 0-10 mois }\end{array}$ \\
\hline 4015 & 2.11 .70 & 25 & 245 & 251 & 220 & 0,733 \\
4020 & 19.11 .70 & 23 & 197 & 239 & 174 & 0,580 \\
4022 & 22.11 .70 & 30 & 196 & 308 & 266 & 0,887 \\
\hline
\end{tabular}

Les résultats des abattages apparaissent au tableau $\mathrm{n}^{\circ}$ VII.

TABLEAU $\mathrm{N}^{\circ} \mathrm{VII}$

Poids avant jeûne

Poids après jeûne

Paurcentage de perte au jeûre

Poids carcasse chaude

Poids carcasse froide

Pourcentage de perte au ressuyage

Rendement

Rendement vrai

Pourcentage contenu de panse

Pourcentage 5ème quartier

Longueur carcasse

Epaisseur cuisse

Epaisseur plat de côtes

Indice de compacité

Conformation carcasse

Gras carcasse

Graisse couverture

Gras de rognon

Graisse musculaire

\begin{tabular}{|c|c|c|}
\hline 4020 & 4015 & 4022 \\
\hline 239 & 251 & 308 \\
217 & 235 & 285 \\
9,20 & 6,37 & 7,46 \\
117,2 & 125,1 & 161,0 \\
114,2 & 122,1 & 157,2 \\
2,55 & 2,39 & 2,36 \\
54,0 & 53,2 & 56,5 \\
59,5 & 58,6 & 61,6 \\
9,21 & 9,23 & 8,31 \\
30,41 & 32,08 & 29,64 \\
102,0 & 101,0 & 105,0 \\
18,4 & 17,6 & 19,8 \\
2,2 & 2,5 & 2,9 \\
1,14 & 1,23 & 1,53 \\
3,5 & 4,0 & 4,5 \\
4,0 & 4,0 & 4,5 \\
4,0 & 4,0 & 4,5 \\
4,5 & 4,5 & 4,5 \\
2,0 & 2,0 & 2,0 \\
\hline
\end{tabular}




\section{Commentaires}

1. Les rendements obtenus sur ces animaux de 11 mois environ sont en moyenne de 54,5 p. 100 , ce qui est une bonne performance. Le rendement vrai moyen est de 60 p. 100.

2. Les carcasses sont d'un poids moyen à peu près similaire $(134,1 \mathrm{~kg})$ à celui des carcasses abattues en moyenne sur la place de Dakar qui pèsent $150 \mathrm{~kg}$ (VALENZA et collab., 1971) et qui proviennent en moyenne d'animaux largement adultes.

3. La conformation de la carcasse est jugée bonne à très bonne. Le profil ischio tarsien est subconvexe en particulier pour la carcasse $n^{0} 4022$.

4. La charge en tissus graisseux est bonne en ce qui concerne la carcasse et le gras de rognon. Sur le plan musculaire, elle est assez faible.

5. La couleur est considérée par les bouchers de la place comme incorrecte car la viande n'est ni rouge, ni blanche, ce qui n'est pas étonnant si l'on considère la composition de la ration. Trois animaux de ce lot seront prochainement abattus à l'âge moyen de 18 mois pour précisément apprécier l'évolution de la couleur de la carcasse en fonction de l'âge.

\section{CONCLUSION}

Les résultats des abattages effectués en 1971 corroborent ceux obtenus en 1970. Chez les animaux nés en 1968 , les rendements sont un peu moins bons que ceux de l'année précédente mais restent cependant excellents. Ces résultats confirment les fortes possibilités de l'animal dans le domaine de l'engraissement.

En ce qui concerne les abattages effectués sur les jeunes animaux de 11 mois, ils montrent qu'à l'aide d'une alimentation telle que celle qui est distribuée, un raccourcissement saisissant de la période d'entretien de l'animal peut être facilement obtenu.

\section{RESUME}

Dans le cadre de l'extériorisation des potentialités bouchères du zébu Gobra, six animaux ont été abattus en 1971. Trois animaux, nés en 1968 et élevés depuis leur naissance à l'aide d'un concentré distribué à volonté, ont donné des carcasses de $381,359,296 \mathrm{~kg}$ avec des rendements de 60,6 , 60,0 et 58 p. 100 respectivement. L'âge moyen était de 39 mois. Les trois jeunes animaux sont nés en 1970 de femelles "exténorisées \% et ont bénéficié du concentré ad libitum en plus de la lactation améliorée de leurs mères. Les abattages ont été pratiqués à 11 mois en moyenne. Les poids des carcasses sont de 161,125 et $117 \mathrm{~kg}$ et les rendements de 56,5 , 53,0 et 54 p. 100 .

\section{SUMMARY}

\section{Phenotypic potentialities of Zebu Gobra}

Six animals of which phenotypic potentialities have been expressed by a rational feeding from birth were slaughtered in october 1971. Three of them, 39 months old showed carcasses of 381,359 and $296 \mathrm{~kg}$ with yields respectively of $60.6-60,0$ and 58 p. 100 .

Three young bulls fed on the same way, and borned from "expressed" cows with an improved lactation were slaughtered at 11 months old. The carcasses weight are 161,125 and $117 \mathrm{~kg}$ with yields of $56,5-53,0$ and 54 p. 100.

\section{RESUMEN}

\section{Exteriorización de los potenciales deI cebú Peulh de Senegal (Gobra)}

Durante el estudio sobre la exteriorızación de los potenciales carniceros del cebú Gobra, se mataron seıs animales en 1971. Tres animales, nacidos en 1968 y alimentados desde su nacimiento con un concentrado distribuido ad libitum, producieron canales de 381,359 y $296 \mathrm{~kg}$ con rendimientos respectivamente de $60,6,60$ et 58 p. 100. Era de 39 meses la edad media. 
Tres jovenes animales nacieron en 1970 de hembras « exteriorizadas » y se añadió concentrado ad libitum en su alimentación constituida por el producto de la lactación mejorada de sus madres. Se mataron los animales a la edad media de 11 meses. Los pesos de las canales fueron de 161, 125 y $117 \mathrm{~kg}$ y los rendimientos de $56,6,53$ y 54 p. 100.

\section{BIBLIOGRAPHIE}

1. CRAPLET (C.), Traité d'élevage moderne, tome VIII, La viande des bovins de l'étable, de l'éleveur à l'assiette du consommateur, livre I, pp. 248-49, Paris, Vigot Frères, 1966.

2. DENIS (J. P.) et VALENZA (J.), Extériorisation des potentialités du zébu Gobra. I. De la naissance au sevrage, Communication au Colloque OCAM sur l'Elevage. Fort-Lamy, décembre 1969.

3. DENIS (J. P.) et VALENZA (J.), Influence du niveau alimentaire sur la croissance du zébu peulh sénégalais (Gobra), Communication au Congrès mondial vétérinaire, Mexico, août 1971.

4. DENIS (J. P.) et VALENZA (J.), Extériorisation des potentialités du zébu peulh sénégalais (Gobra), Rev. Elev. Méd. vét. Pays trop., 1971, 24 (3) : 409-18.

5. DUBOSE (L. E.), CARTWRIGHT (T. C.) et COOPER (R. J.), Diedicting steak and roast meat from production and carcass traits, J. Anim. Sci., 1967, 26 : 688-93.

6. DUMAS (R.) et LHOSTE (Ph.), Variations du poids vif et du rendement en viande de boufs zébus de l'Adamaoua au cours de Ia saison sèche, Rev. Elev. Méd. vét. Pays trop., 1966, 19 (4): 573-79.

7. EL SHAFIE (S. A.), Fattening of Sudan Zebu cattle. I. Weight gain and carcass analysis of castrated and non castrated Butana calves, Sudan J. vet. Sci. Anim. Husb., 1965, 6 (1) : 33-40.

8. GILIBERT (J.), Le contrôle de la valeur bouchère des bovins. Rapport de stage. I.E.M.V.T. no 4/Doc. 24, I, 66.

9. GILIBERT (J.), Valeurs bouchères des zébus à Madagascar, Rev. Elev. Méd. vét. Pays trop., 1971, 24 (3): 445-65.

10. HALL, Note sur les rendements en carcasse et les pourcentages d'os du bétail soudanais. Sudan J. vet. Sci. Anim. Husb., 1962, 3 (1).
11. HARTE (F. J.) et CONNIFFE (D.), Studies on cattle of varying growth potential for beef production. I. Growth rate, feed conversion efficiency, carcass yield and offals. II. Carcass composition and distribution on "lean meat", fat and bone, Ir. J. agr. Res., 1967, 6: 137-52, 153.70.

12. HEWETSON (R.W.), Difference in muscle: bone ratio between zebu cross and british breed steers, Proc. Aust. Soc. Anim. Prod., 1970, 8 : 100-105.

13. LETROTEUR (R.), Rendements comparés en muscles consommables, os, graisse, aponévroses après transformation classique (coupe de Paris d'une demi-carcasse de N'Dama et de Zébu), Rev. Elev. Méd. vét. Pays trop., 1957, $10: 41-44$.

14. La race charolaise - Herd Book charolais, 1962.

15. ROSSI (G.), Fattening tests with young crossbred bulls, Brown Alpine x Modica Sardinian (Ital.) Alimentaz Anim., 1967, 11: 497-509; in : Anim. Breed. Abstr., 1968, 36 (2): 198.

16. SCHMITTER (W.), AVERDUNK (G.), MATZKE (P.), PILZWEGER (J.), RUPP (K.), MITTELSTADT (W.), The carcass composition of 500-dayold German Simmental bulls progeny-tested in Bavaria (Allemand), Bayer landw. Jb. 47: 811-18. In : Anim. Breed Abstr., 1968, 36 (2): 198.

17. VALENZA (J.), DENIS (J. P.), DIALLO (M.S.) et THIONGANE (A.I.), La viande au Sénégal, production et hygiène - perspectives, Communication aux VIIE Journées Médicales de Dakar, janvier 1971.

18. VALENZA (J.), CALVET (H.) et ORUE (J.), Essais d'engraissement intensif de zébus peulh sénégalais (Gobra). Dakar-Hann, I.E.M.V.T., Laboratoire national de l'Elevage, février 1970 , $36 \mathrm{p}$. 


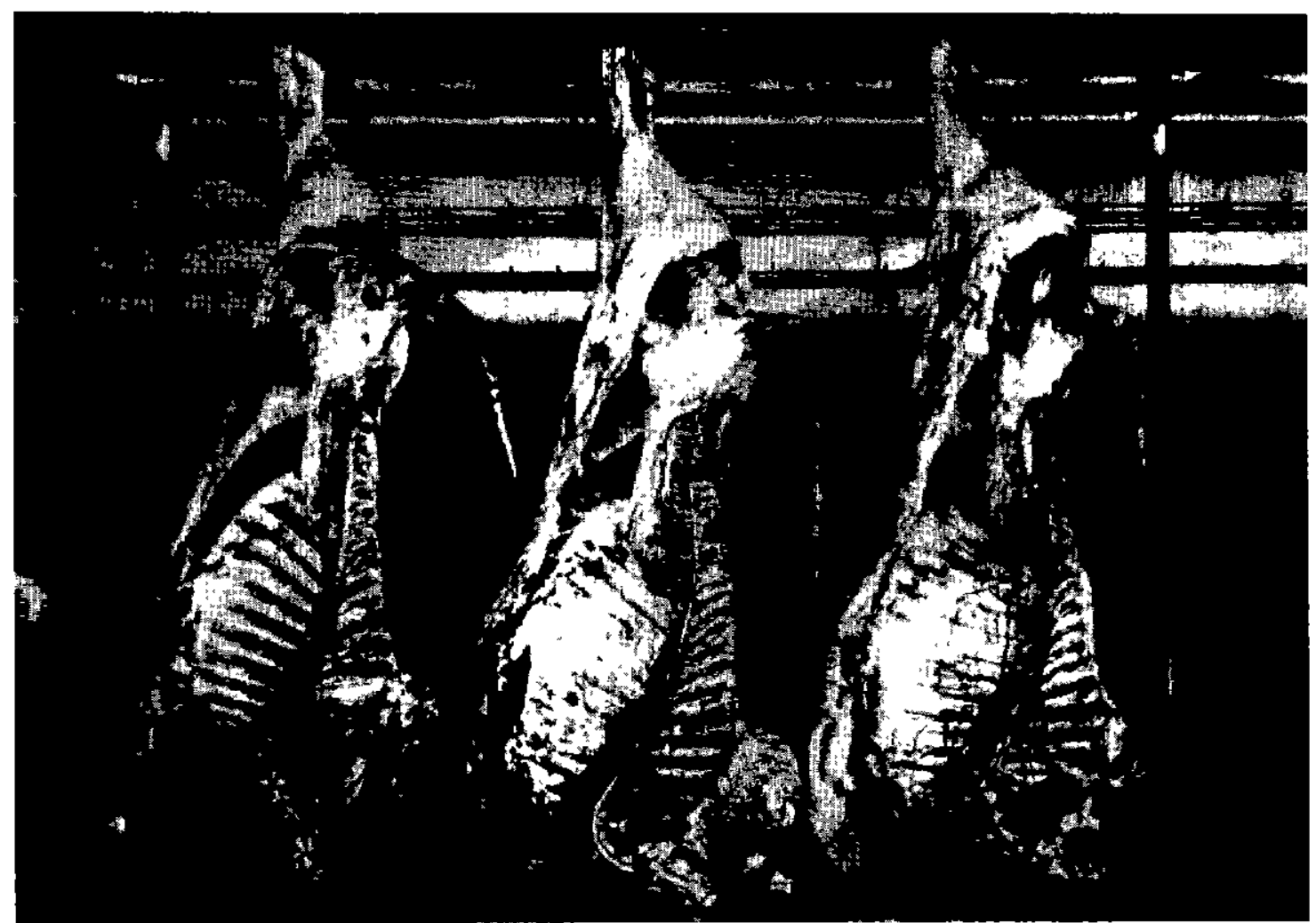

Photo 1. - Carcasses des taureaux.

De gauche à droite: gains minimal, moyen et maximal.

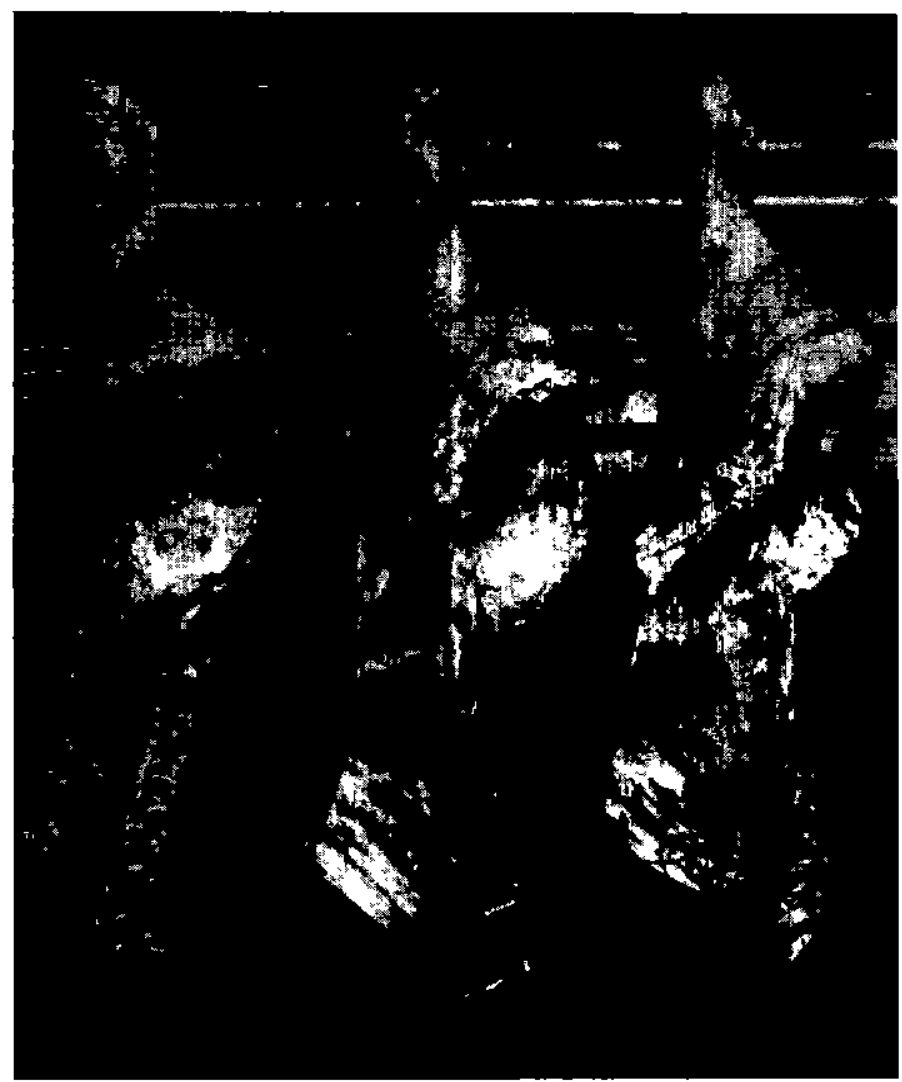

Photo 2. - Pans des taureaux.

De gauche à droite :

gains minimal, moyen et maximal. 


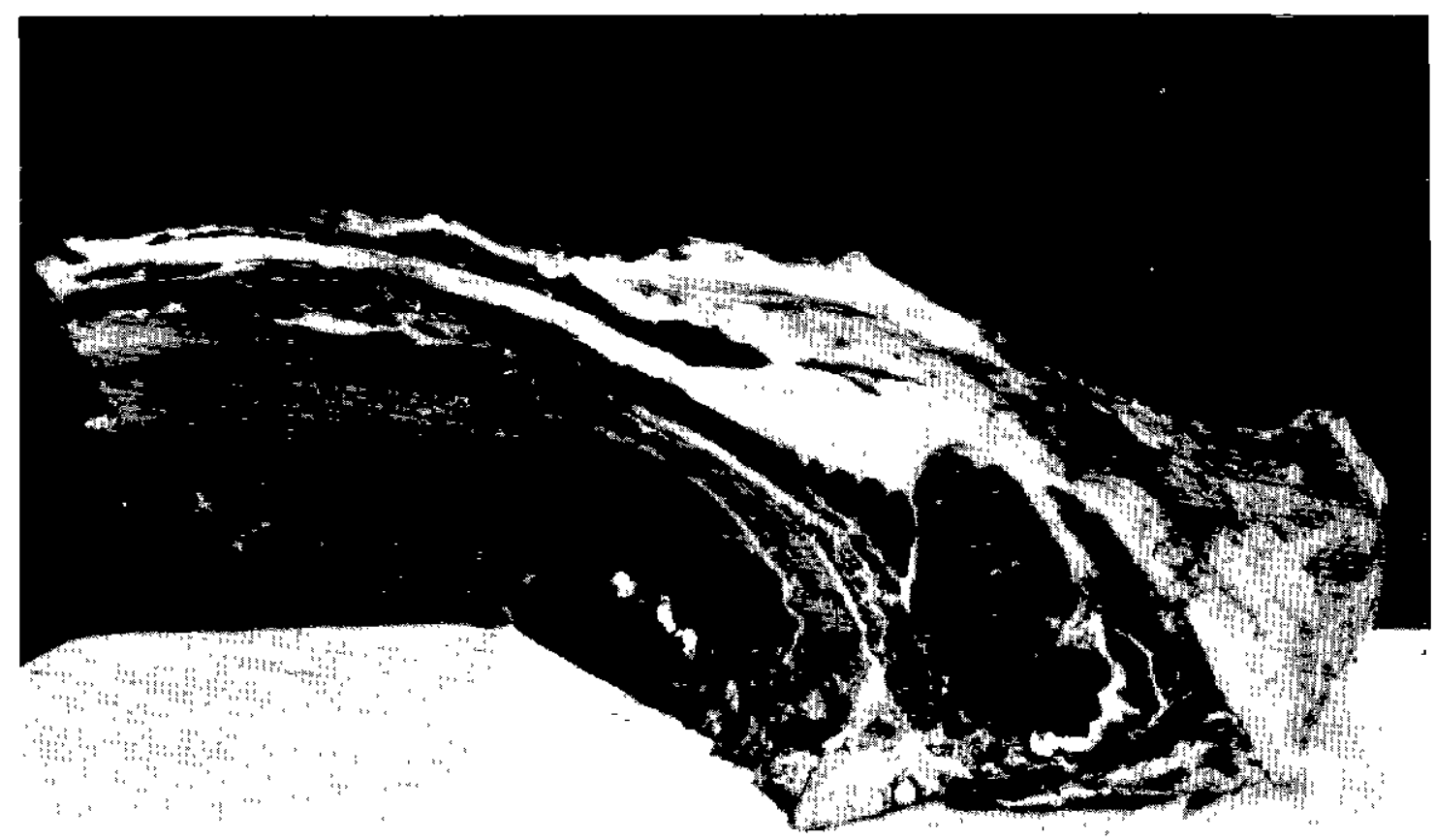

Photo 3. - Train de côtes taureau gain maximal; coupe au ge espace jntercostal.

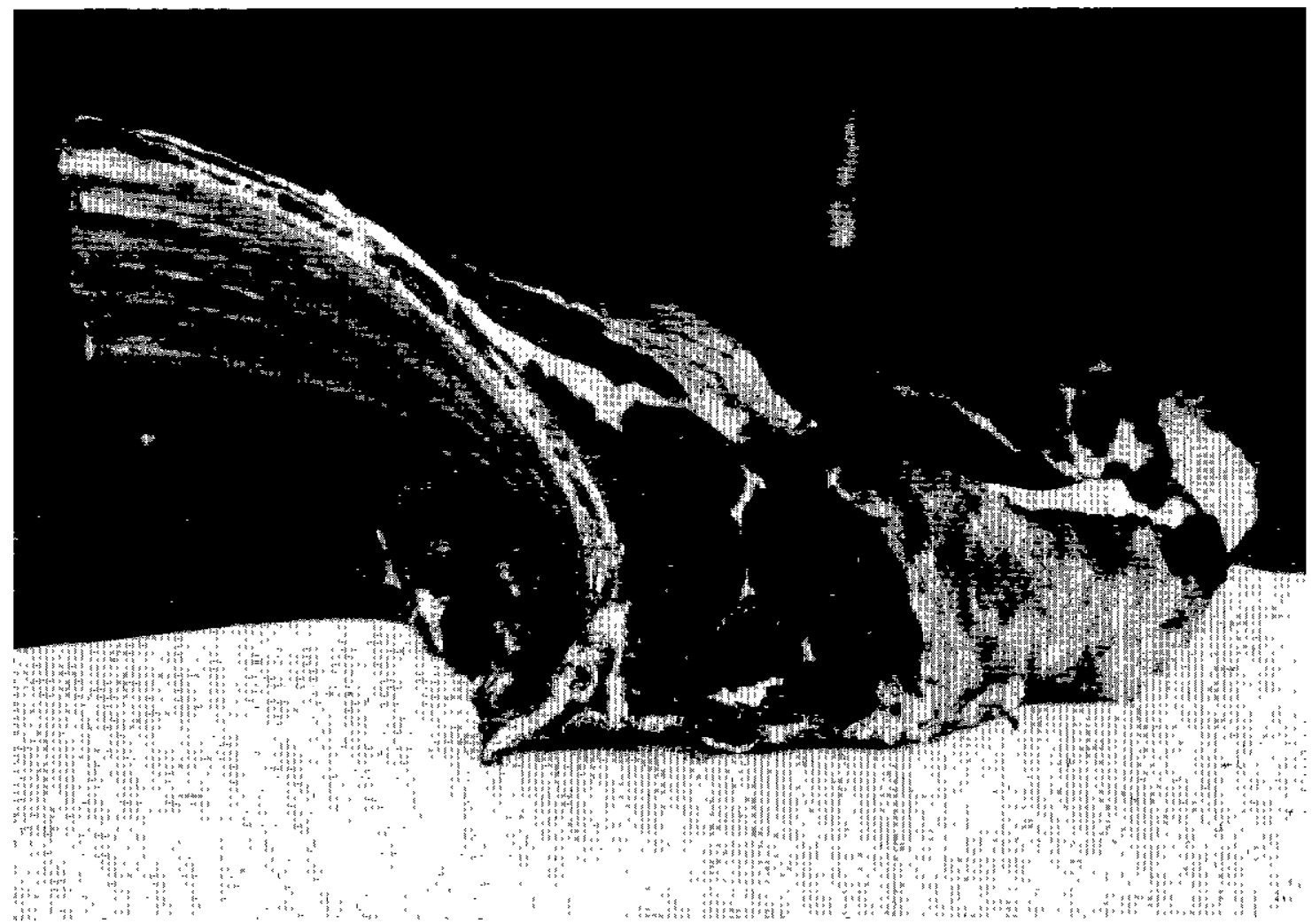

Photo 4. - Train de côtes taureau gain minimal; coupe au ge espace intercostal. 


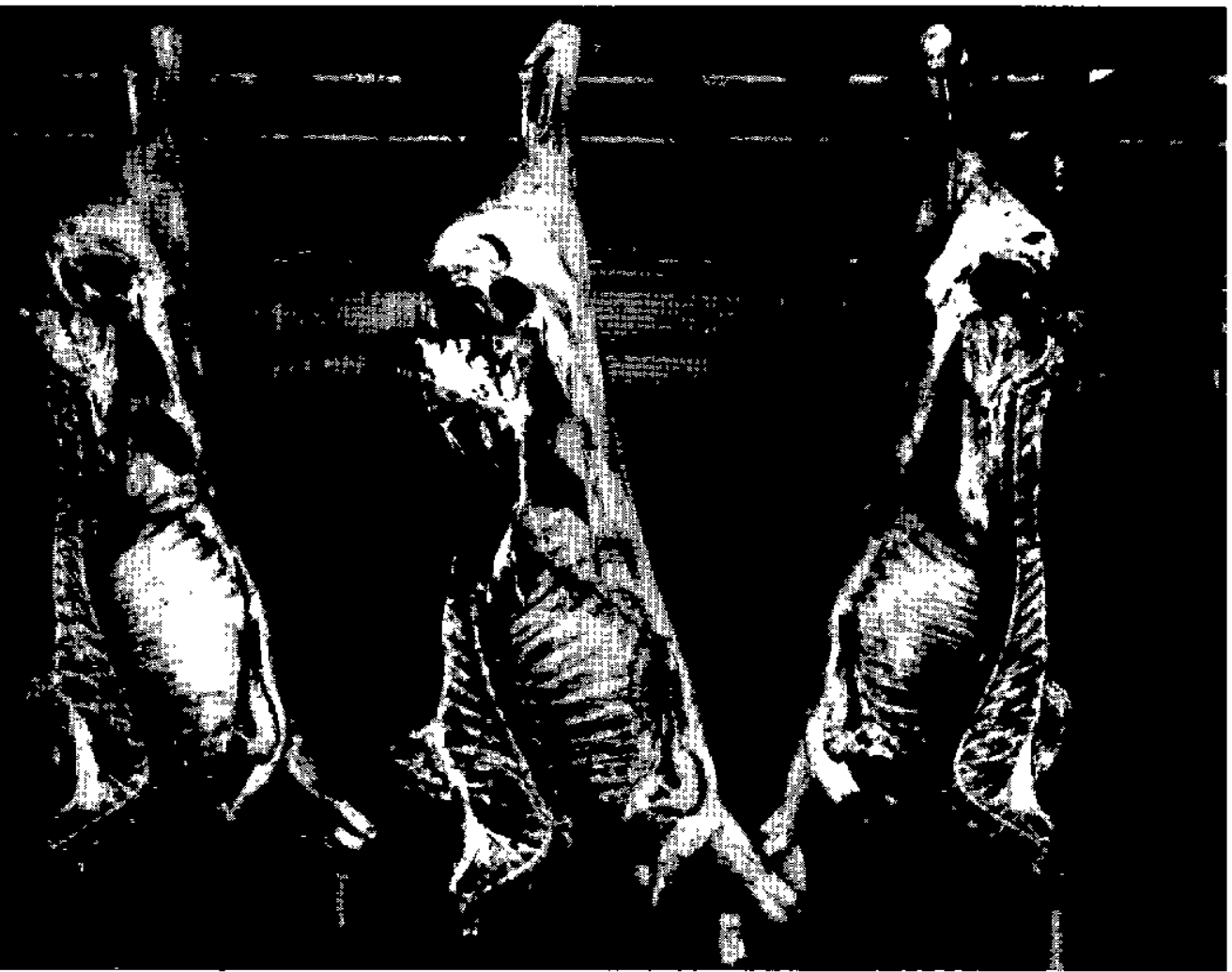

Photo 5. - Carcasses de taurillons 11 mois.

De gauche à droite: gains minimal, maximal et moyen.

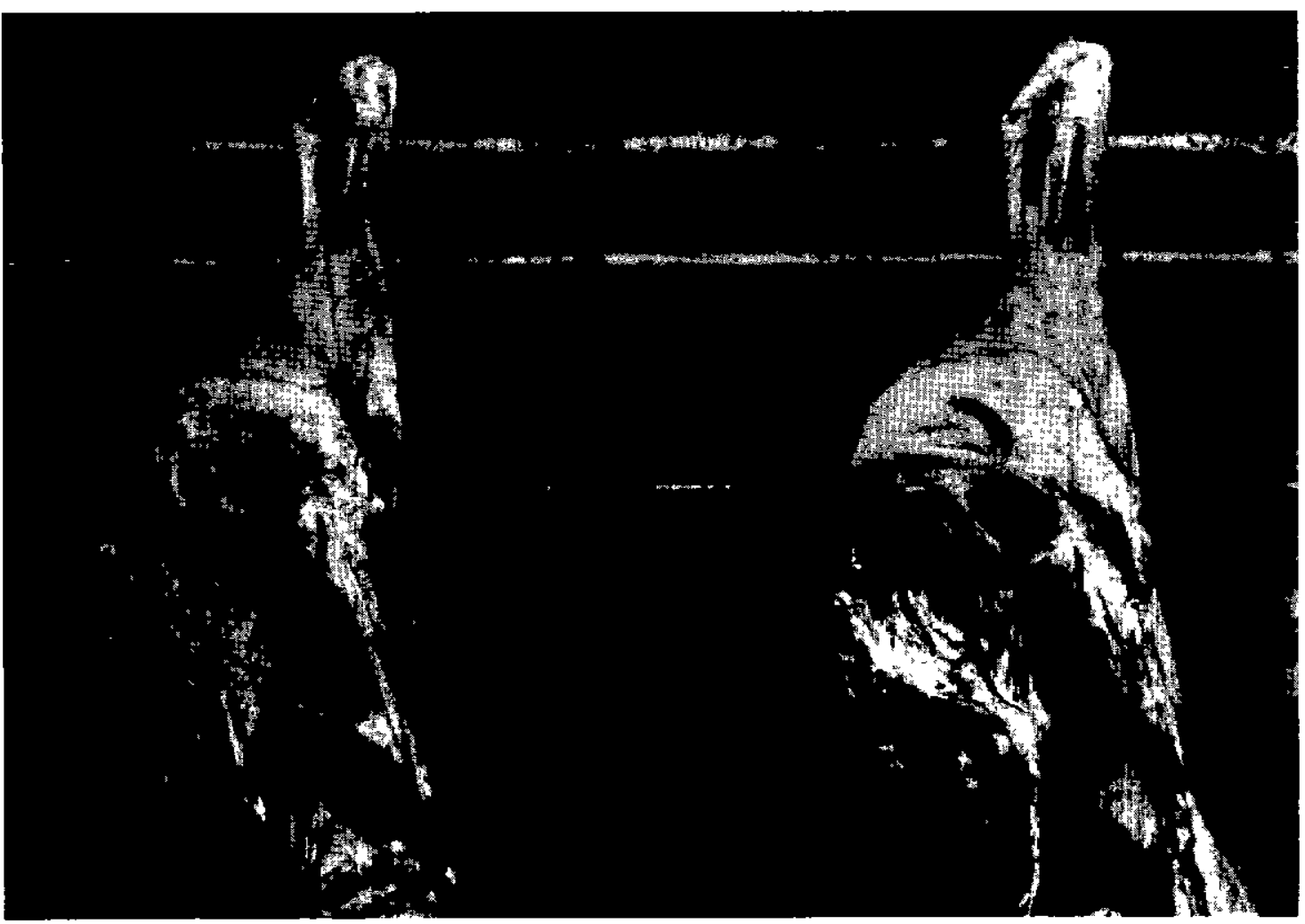

Photo 6. - Globes de taurillons 11 mois.

De gauche à droite: gains minimal et maximal. 